\title{
Study of Excimer Laser Irradiation Effects on Properties of Crystalline YBaCuO Thin Films
}

\author{
A. Abal'oshev ${ }^{a}$, I. Abal'osheva ${ }^{a}$, S.J. LeWAndowski ${ }^{a}$, \\ C.J. VAN DER BeEK ${ }^{b}$, M. KONCZYKOWSKI ${ }^{b}$, G. RIzZA $^{b}$ \\ AND S. BARBANERA ${ }^{c}$ \\ ${ }^{a}$ Instytut Fizyki PAN, al. Lotników 32/46, 02-668 Warszawa, Poland \\ ${ }^{b}$ Laboratoire des Solides Irradiés, CNRS-UMR 7642 and CEA/DSM/DRECAM \\ Ecole Polytechnique, 91128 Palaiseau Cedex, France \\ ${ }^{c}$ Istituto di Fotonica e Nanotecnologie - CNR \\ Via Cineto Romano 42, 00156 Roma, Italy
}

The influence of UV laser irradiation on the physical properties of epitaxial $\mathrm{YBa}_{2} \mathrm{Cu}_{3} \mathrm{O}_{7-\delta}$ thin $(<1 \mu \mathrm{m})$ films fabricated by laser ablation was studied. The samples were irradiated by pulsed excimer laser beam at different incident energy densities $E_{\mathrm{ir}} \leq 130 \mathrm{~mJ} / \mathrm{cm}^{2}$, i.e. $2-3$ times below the ablation threshold of $\mathrm{YBaCuO}$. The analysis of the sample cross-section using transmission electron microscope shows that such irradiation forms a disordered layer on the top of the crystalline film. Sample surface amorphization was observed also by atomic force microscope. Due to strong UV irradiation absorption in $\mathrm{YBaCuO}$ this surface layer acts like a protective cover for the sample interior. Only a small reduction of superconducting critical temperature $T_{\mathrm{c}}$ was observed. Both transport measurements in normal state and magneto-optical studies in superconducting state indicate that between the disordered layer and the bottom non-modified part of the film there exists a wide transitional region with reduced carrier concentration.

PACS numbers: 74.25.Fy, 74.72.Bk, 74.78.Bz, 81.15.Fg

\section{Introduction}

High energy laser irradiation of high-temperature superconductor (HTSC) materials can lead to nonreversible modifications of their physical properties [1]. 
In the case of thin films (thickness $<1 \mu \mathrm{m}$ ), irradiation by IR or visible sources induces structural transformations in the whole sample volume $[2,3]$. The influence of UV irradiation is limited to a near-surface region of film due to the short absorption length $d=44 \mathrm{~nm}$ in this spectral range [4]. This phenomenon can be used to form e.g. a thin dielectric passivation layer on the top of the thin film superconducting structure.

\section{Experimental}

In this paper we present a brief overview of our investigation of the effects of laser irradiation on the properties of epitaxial $\mathrm{YBa}_{2} \mathrm{Cu}_{3} \mathrm{O}_{7-\delta}$ thin films obtained by laser ablation from stoichiometric " 123 " target. The films were deposited on $5 \times 5 \mathrm{~mm}^{2}$ single crystalline substrates at heater temperature $785^{\circ} \mathrm{C}$ and at $280 \mathrm{mTorr}$ oxygen pressure. The excimer $\mathrm{KrF}$ (wavelength $\lambda=248 \mathrm{~nm}$ ) laser with the pulse duration $\tau=28 \mathrm{~ns}$, repetition rate $10 \mathrm{~Hz}$ and energy density $1.5 \mathrm{~J} / \mathrm{cm}^{2}$ at the target surface was used. After deposition, the samples were cooled down to room temperature with the rate of $-20 \mathrm{~K} / \mathrm{min}$ at 300 Torr oxygen pressure, and patterned, as required, for resistance, the Hall effect, and magnetooptical measurements using conventional UV photolithography and wet-etching process. The samples were irradiated by $\mathrm{KrF}$ and $\mathrm{XeCl}(\lambda=308 \mathrm{~nm}, \tau=28 \mathrm{~ns})$ lasers at energy density $E_{\mathrm{ir}} \leq 130 \mathrm{~mJ} / \mathrm{cm}^{2}$ on film surface, which was close to the vaporization (etching) threshold $E_{\text {eth }}=110 \mathrm{~mJ} / \mathrm{cm}^{2}$ [4] and was 2-3 times below the ablation threshold $E_{\text {th }}=0.3-0.4 \mathrm{~J} / \mathrm{cm}^{2}$ of $\mathrm{YBaCuO}$ [5]. Irradiation was carried out in ambient conditions.

\section{Results and discussion}

The atomic force microscope (AFM) images (left panel of Fig. 1) show that laser annealing causes partial melting of defects on the film top (like outgrows) and remarkably reduces the surface roughness. On the other hand, the cross-section transmission electron microscope (TEM) image (right panel of Fig. 1) of irradiated film gives direct evidence that at high laser fluence the surface layer of thin film becomes amorphous. This "A" region appears to be separated from the crystalline part "C" of the film by a sharp boundary.

A fast increase in the amorphous surface layer thickness at the beginning of the laser treatment produces a marked growth of sample resistance $R(N)$, where $N$ is the number of consecutive laser pulses (Fig. 2a). Due to a strong absorption of $\mathrm{UV}$ irradiation in $\mathrm{YBaCuO}$ [4], the surface layer considerably reduces the impact of laser light on film interior. As a result, the dependence of $R$ on the irradiation dose becomes weaker (at least at $E_{\mathrm{ir}}<E_{\text {eth }}$ ) and the crystalline $\mathrm{YBaCuO}$ thin film remains superconducting even after several hundreds of laser shots (Fig. 2b).

UV irradiation increases both film normal state resistance $R=\rho_{a b} l w t$ and the Hall voltage $U_{\mathrm{H}}=R_{\mathrm{H}} I H_{z} / t$ (see Fig. 2c), where $l, w$, and $t$ are the length, 


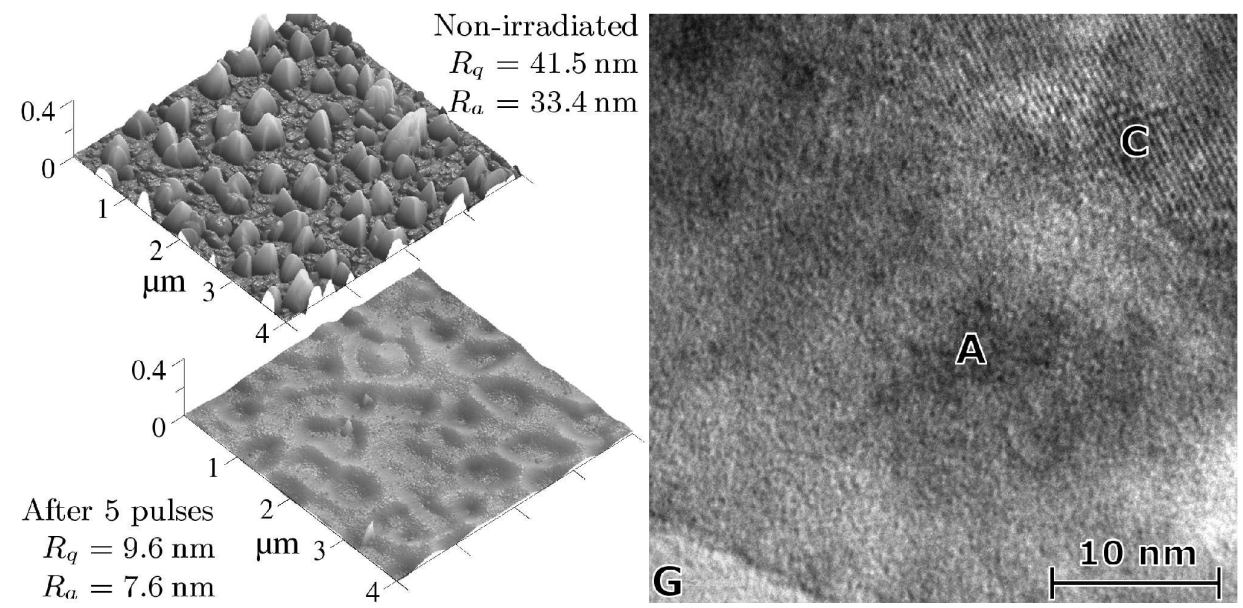

Fig. 1. Left panel: AFM images of surface of $100 \mathrm{~nm}$ thick $\mathrm{YBaCuO}$ film deposited on $\mathrm{SrTiO}_{3}$ substrate before and after irradiation by XeCl laser at energy density $E_{\mathrm{ir}}=100 \mathrm{~mJ} / \mathrm{cm}^{2} . R_{q}$ and $R_{a}$ are roughness average and RMS value of the thickness fluctuation, respectively. Right panel: TEM photograph of cross-section of the $200 \mathrm{~nm}$ thick YBaCuO film grown on SAT(70\%)-CAT(10\%)-LA(20\%) substrate and irradiated by three pulses of $\mathrm{KrF}$ laser at $E_{\mathrm{ir}}=100 \mathrm{~mJ} / \mathrm{cm}^{2}$ per pulse. Amorphous and crystalline parts of the film are denoted as (A) and (C), respectively, the layer of glue on the sample surface - as $(G)$.

width, and thickness of the sample, respectively, $\rho_{a b}$ is the longitudinal resistance, $R_{\mathrm{H}}$ is the Hall coefficient, $I$ is the measurement current, and $H_{z}$ is the external magnetic field (in the considered case $H_{z}=0.5 \mathrm{~T}$ ). Assuming that changes of $R$ and $U_{\mathrm{H}}$ are caused only by the reduction of $t$, we can expect that the following expression is true:

$$
\frac{R^{\mathrm{ir}}(T)}{R(T)}=\gamma \frac{U_{\mathrm{H}}^{\mathrm{ir}}(T)}{U_{\mathrm{H}}(T)}, \quad \gamma=1,
$$

where $R^{\mathrm{ir}}$ and $U_{\mathrm{H}}^{\mathrm{ir}}$ relate to the irradiated sample. However, for sample $1 \gamma=1.3$ and for sample $2 \gamma=1$.5. It means that a wide transitional region with reduced carrier concentration (possibly oxygen-depleted) was formed under the amorphous top layer. Temperature dependence of the Hall angle $\theta_{\mathrm{H}}\left(\tan \left(\theta_{\mathrm{H}}\right)=R_{\mathrm{H}} H_{z} / \rho_{a b}\right)$ shown in Fig. 2d for both virgin and irradiated samples has a typical form for HTSC materials proposed by Anderson [6]: $\cot \left(\theta_{\mathrm{H}}\right)=A T^{2}+B$, where $A$ and $B$ are constants. Growth of both $A$ and $B$ corresponds to decreasing oxygen concentration in the sample [7] and to increasing concentration of point defects in $\mathrm{Cu}-\mathrm{O}$ planes of $\mathrm{YBaCuO}[8]$.

Measurements of magnetic moment distribution in the superconducting films at various temperatures, from 10 to $70 \mathrm{~K}$, and in the external magnetic fields up to 350 Gs were realized by means of a ferromagnetic iron-garnet indicator, 

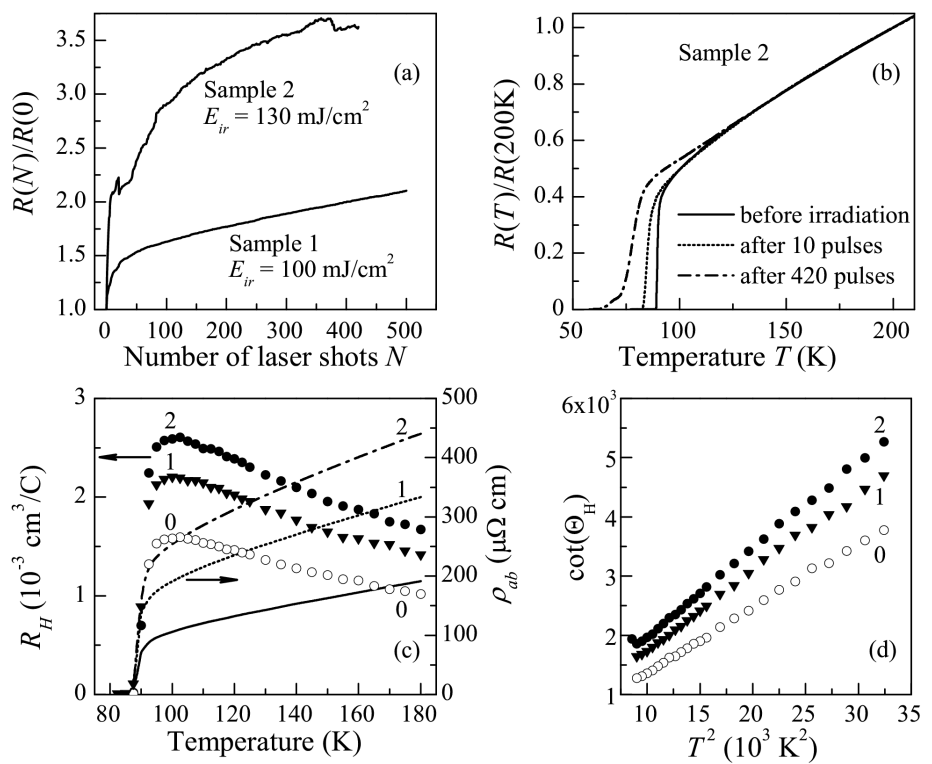

Fig. 2. (a) Normalized sample resistance $R$ as a function of number of $\mathrm{KrF}$ laser pulses $N$; (b) resistance vs. temperature characteristics of $\mathrm{YBaCuO}$ thin film deposited on SAT-LA for different irradiation doses; temperature dependences of the Hall coefficient, resistivity (c) and the Hall angle (d) for virgin film grown on $\mathrm{SrLaGaO}_{4}$ and for samples irradiated by 1 and 2 pulses of $\mathrm{KrF}$ laser at $E_{\text {ir }}=100 \mathrm{~mJ} / \mathrm{cm}^{2}$.
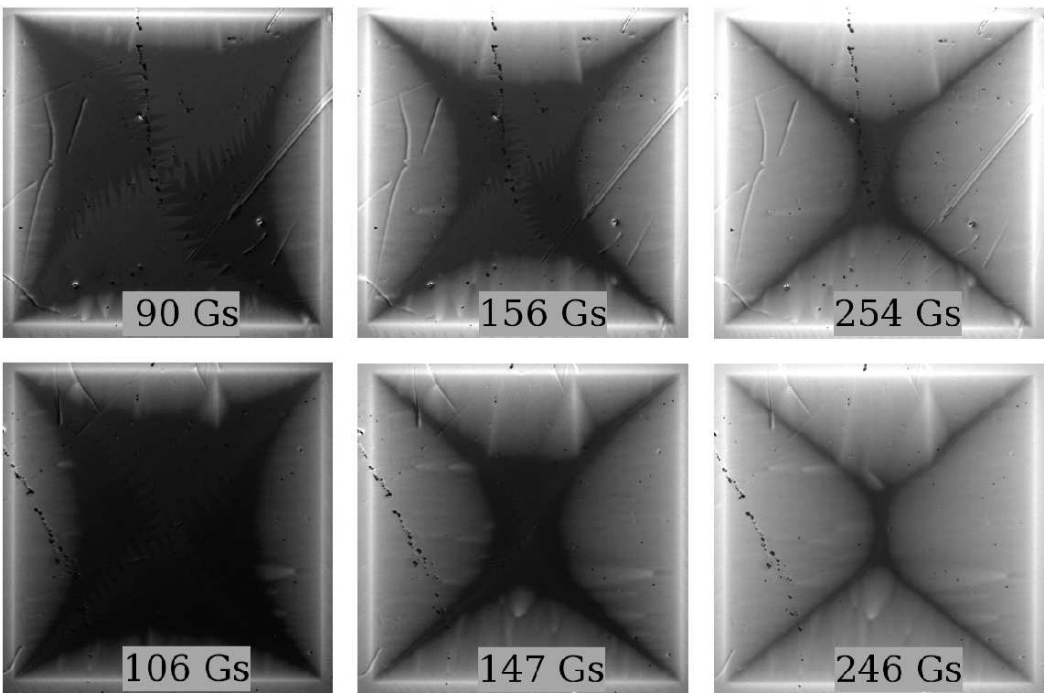

Fig. 3. Visualization of magnetic field penetration in virgin (top 3 images) and laser treated $\mathrm{YBaCuO}$ film at $20 \mathrm{~K}$ (bottom row). The $200 \mathrm{~nm}$ thick film was deposited on SAT-CAT-LA substrate and irradiated by 3 pulses of $\mathrm{KrF}$ laser at $100 \mathrm{~mJ} / \mathrm{cm}^{2}$. 
which rotates the plane of polarization of linearly polarized light proportionally to the local magnetic field (Faraday effect). Images of polarized light intensity corresponding to the magnetic field distribution were obtained in such manner (Fig. 3). Magnetic field penetrates the laser treated film and escapes from it more easily than in the case of a non-irradiated sample. It means that laser treatment of the film leads to a critical current $I_{\mathrm{c}}$ decrease in the irradiated areas. The decrease in superconducting layer thickness should lead to an equivalent reduction of the absolute value of $I_{\mathrm{c}}$. In the future, we plan to use the magneto-optical images in order to calculate $I_{\mathrm{c}}$, and to use these data to estimate the thickness of the non-modified layer.

\section{Conclusions}

To conclude, our results indicate that high energy density UV laser irradiation forms an amorphous surface layer followed by an oxygen-depleted region in crystalline $\mathrm{YBaCuO}$ thin films. The film region close to the substrate remains non-modified.

\section{Acknowledgment}

This work was supported by the State Committee for Scientific Research (Poland) grants 2P 03B 04423 and 4T 11B 02625.

\section{References}

[1] S.J. Lewandowski, G. Jung, V.D. Okunev, Z.A. Samoilenko, V.A. Isaev, A. Abal'oshev, P. Gierłowski, A. Klimov, S. Barbanera, Proc. SPIE 4086, 518 (2000); V.D. Okunev, Z.A. Samoilenko, A. Abal'oshev, I. Abal'osheva, P. Gierłowski, A. Klimov, S.J. Lewandowski, V.N. Varyukhin, S. Barbanera, Phys. Rev. B 62, 696 (2000).

[2] S. Otsubo, T. Maeda, A. Morimoto, T. Shimizu, Y. Minamikawa, Y. Yonezawa, Jpn. J. Appl. Phys. 28, 2211 (1989).

[3] N. Aizaki, K. Terashima, J. Fujita, S. Matsui, Jpn. J. Appl. Phys. 27, L231 (1988).

[4] A. Inam, X.D. Wu, T. Venkatesan, S.B. Ogale, C.C. Chang, D. Dijkkamp, Appl. Phys. Lett. 51, 1112 (1987).

[5] R.K. Singh, J. Narayan, Phys. Rev. B 41, 8843 (1990).

[6] P.W. Anderson, Phys. Rev. Lett. 67, 2092 (1991).

[7] A. Carrington, D.J.C. Walker, A.P. Mackenzie, J.R. Cooper, Phys. Rev. B 48, 13051 (1993).

[8] T.R. Chien, Z.Z. Wang, N.P. Ong, Phys. Rev. Lett. 67, 2088 (1991). 